\title{
Insights from intermittent binocular rivalry and EEG
}

\author{
Michael A. Pitts ${ }^{1 *}$ and Juliane Britz ${ }^{2}$ \\ 1 Department of Psychology, Reed College, Portland, OR, USA \\ ${ }^{2}$ Department of Fundamental Neuroscience, University of Geneva, Geneva, Switzerland
}

Edited by:

Naotsugu Tsuchiya, RIKEN, Japan

Reviewed by:

Philipp Sterzer, University Hospital

Charité, Germany

Jason Forte, The University of

Melbourne, Australia

*Correspondence:

Michael A. Pitts, Department of

Psychology, Reed College, 3203 SE

Woodstock Blvd, Portland, OR

97202-8199, USA.

e-mail:mpitts@reed.edu
Novel stimulation and analytical approaches employed in EEG studies of ambiguous figures have recently been applied to binocular rivalry. The combination of intermittent stimulus presentation and EEG source imaging has begun to shed new light on the neural underpinnings of binocular rivalry. Here, we review the basics of the intermittent paradigm and highlight methodological issues important for interpreting previous results and designing future experiments. We then outline current analytical approaches, including EEG microstates, event-related potentials, and statistically based source estimation, and propose a neural model of the sequence of brain events that may underlie different aspects of binocular rivalry. Finally, we discuss the advantages and limitations of using binocular rivalry as a tool to investigate the neural basis of perceptual awareness.

\section{Keywords: binocular rivalry, perceptual reversals, EEG microstates, ERP, visual awareness}

\section{INTRODUCTION}

Traditionally, binocular rivalry involves continuous (uninterrupted) presentation of different stimuli to the left and right eyes. Perception stochastically alternates between these two stimuli every few seconds (Tong et al., 2006; Sterzer et al., 2009) and is likely to involve competition between neural signals representing each stimulus and its associated percept. (Blake and Logothetis, 2002). fMRI studies in humans have identified activity in frontal and parietal areas in addition to early visual areas during binocular rivalry (Lumer et al., 1998; Lumer and Rees, 1999). Single-cell recordings in non-human primates have shown that firing rates of cells in higher-level visual areas (in the ventral stream) are correlated with the momentarily active percept (Leopold and Logothetis, 1996; Logothetis et al., 1996). These measures provide complementary spatial/temporal information: $\mathrm{fMRI}$ provides wide spatial coverage (i.e., the entire brain) but with low temporal resolution, whereas single-cell recordings provide high temporal resolution but measure activity at a limited number of spatial locations at any given time. Here, we review an approach that affords measures with concurrently high temporal resolution and whole brain spatial coverage: EEG source imaging (Michel et al., 2009). Studies that have used this analysis approach in combination with intermittent stimulation have begun to provide fresh insights into the sequence of neural events that contribute to different aspects of binocular rivalry (Pitts et al., 2010; Britz et al., 2011; Britz and Pitts, 2011).

\section{THE INTERMITTENT PARADIGM}

In all binocular rivalry paradigms subjective reports are required in order to relate measured brain activity to each percept or to transitions between percepts. The subjective reports (usually keypresses) are used to indicate the current percept and to index the time at which a perceptual transition has occurred. With continuous rivalry, the time intervals between the perceptual changes themselves and the reports of such changes are likely to vary from trial-to-trial by tens to hundreds of milliseconds. For fMRI, this temporal jitter between percept and report is unlikely to affect measurements of brain activity which are on the scale of several seconds. With EEG however, such trial-to-trial variation can obliterate event-related potentials (ERPs) and thus compromise the advantages offered by this temporally precise measure.

To alleviate this problem, stimuli can be presented intermittently (alternated with blank intervals) and EEG recordings can be time-locked to stimulus onset instead of subjects' reports. Influenced by previous work (Orbach et al., 1963, 1966; O'Donnell et al., 1988), Kornmeier and Bach (2004) were the first to detail the advantages of the intermittent paradigm for the measurement of ERPs during ambiguous figure perception. An ERP component dubbed the "reversal negativity" (RN) was found to be associated with perceptual changes reported by subjects while viewing the Necker cube (Kornmeier and Bach, 2004). The RN component, which is characterized by a negative amplitude shift for perceptual reversals at $\sim 200-300$ ms post-stimulus onset over the posterior scalp, has subsequently been identified in a variety of studies using various types of bistable stimuli including binocular rivalry (Kornmeier and Bach, 2004, 2005; Kornmeier et al., 2007; Pitts et al., 2007, 2009; Britz et al., 2009; Intaite et al., 2010; Britz and Pitts, 2011). In addition to the RN, an earlier component, the "reversal positivity" (RP; $\sim 100-130 \mathrm{~ms}$ ), and a later component, the "late positive complex" (LPC; 400-600 ms), have also been linked with perceptual reversals (Kornmeier and Bach, 2005; Pitts et al., 2007; Britz et al., 2009; Britz and Pitts, 2011).

Importantly, intermittent rivalry appears to closely resemble continuous rivalry with respect to perceptual dominance periods and reversal rates (Britz et al., 2009; Britz et al., 2011), although to achieve this consistency, the duration of the stimulus and the duration of the intervening blank interval must be fine-tuned. If the stimulus duration is too long, reversals may occur within a single presentation. If the intervening blank interval is too long, reversals can be prevented altogether (Leopold et al., 2002; Sterzer and Rees, 2008). Conversely, if the stimulus duration or blank interval is too brief, subjects will have trouble reporting their percepts during each trial and the resulting ERPs (including motor potentials) for 
a given trial will overlap and thus contaminate ERPs elicited in the subsequent trial. With these concerns in mind, most studies have presented stimuli for durations of $600-800 \mathrm{~ms}$, with 400 600 ms blank intervals. Recently, Brascamp et al. (2009) showed that reversal rates during intermittent rivalry become substantially slower than continuous rivalry when the blank interval is extended beyond $\sim 1.5 \mathrm{~s}$. Kornmeier et al. (2007) systematically manipulated blank interval durations (ranging from $\sim 15-400 \mathrm{~ms}$ ) and found that reversal rates for the Necker cube were reduced for very short blank intervals (e.g., $\sim 15$ and $\sim 50 \mathrm{~ms}$ ). Taken together, previous findings suggest that a "sweet spot" may exist between long and short blank interval durations in which reversal rates for intermittent and continuous rivalry can be made equivalent. However, a systematic manipulation of blank interval durations ranging from very short (e.g., <100 ms) to very long (e.g., >2 s) has not yet been carried-out, and even though reversal rates can be made equivalent, it is not yet clear whether the neural systems supporting perceptual rivalry under each type of presentation paradigm are exactly the same.

Perhaps the most surprising feature of the intermittent paradigm is that perceptual reversals seem to be tightly time-locked to stimulus onset (Orbach et al., 1963). In other words, as the stimuli flash on-and-off, subjects perceive one of the two possible images for a few trials, and then suddenly they experience the alternative percept on the next flash. Subjectively, it is difficult to tell whether the change is perceptual or physical. In fact, subjects often express disbelief that the same stimulus is being flashed on-and-off, insisting that the experimenter must be physically alternating the images on the computer screen. At first glance, this feature of the intermittent paradigm may seem to imply that reversals are exogenously, rather than endogenously, controlled. However, the purpose of the intermittent design is to allow control over when reversals can occur but not whether they actually do occur. Whether a reversal occurs or not in the intermittent paradigm (at least for studies in which appropriately tailored blank interval durations are employed) is determined by the same statistical properties (log normal and gamma distributions) as in continuous rivalry.

\section{WHAT CONDITIONS TO COMPARE AND WHY?}

In the basic intermittent paradigm, subjects are instructed to report whether their perception has changed (or remained the same) on the current trial relative to the previous trial (Kornmeier and Bach, 2004). These reports allow comparisons between ERPs elicited during endogenous "reversals" versus "stability." This paradigm can be extended by adding a "physical alternation" or replay condition in which unambiguous (Kornmeier and Bach, 2004) or non-rivaling stimuli (Lumer et al., 1998; Pitts et al., 2010) are presented at rates that match those of endogenous reversals. This extended design allows additional comparisons between endogenously versus exogenously generated perceptual reversals.

By modifying how subjects report their percepts, a third (and fourth) type of comparison is possible. Instead of having subjects report perceptual changes, subjects can report whether they perceive image A or image B during each trial. This allows additional comparisons between brain activity associated with percept A versus percept B (Pitts et al., 2010). Notably, with this reporting method, the same data can be re-categorized to allow comparisons between reversals and stability. Thus, by instructing subjects to report their percepts (A or B) during binocular rivalry and physical alternation conditions, the same data can be used to make all four types of comparisons: endogenous percept A versus B; exogenous percept A versus B; endogenous reversal versus stability; exogenous reversal versus stability (see Figure 1).

With the goal of comparing ERPs associated with percept A versus $\mathrm{B}$, it is important to choose stimuli that differ on at least one dimension that will produce measurable differences when these stimuli are presented in physical alternation. For example, if ERPs elicited by grating stimuli oriented at $45^{\circ}$ versus $135^{\circ}$ do not differ during physical alternation, it is unlikely that ERPs associated with percepts of these gratings will differ during binocular rivalry. To circumvent this issue, in a recent study we presented gratings of high versus low spatial frequencies (SF) during binocular rivalry and during physical alternation. ERPs were already known to differ considerably for high versus low SF stimuli, so the question was whether (and when) ERPs might differ based on percepts of high versus low SF during rivalry. We found that the amplitude of the $\mathrm{C} 1$ component $(60-100 \mathrm{~ms})$, which is known to be generated in early visual cortex (V1/V2/V3), differed between the two stimuli during physical alternation but not between the two percepts during rivalry, while subsequent ERPs (130-160 ms), with similar scalp distributions as the $\mathrm{C} 1$, differed according to the reported percept in both conditions (Pitts et al., 2010). This pattern of results suggested that the same anatomically early visual areas may play different roles in the rivalry process during different time windows. This strategy, i.e., rivaling stimuli that are known to produce reliable ERP differences when presented in physical alternation, is likely to work for face/house rivalry, upright/inverted face rivalry, as well as the ambiguous face/vase stimulus. Future studies may consider adopting this strategy to help determine the timing and brain regions involved in the resolution of different types of perceptual rivalry.

\section{EEG MICROSTATES AND SOURCE ANALYSES}

While analysis of post-stimulus ERPs under the intermittent paradigm has proven fruitful, EEG microstates can be used to compare brain activity during pre-stimulus as well as post-stimulus time periods. EEG microstates refer to brief $(\sim 80-120 \mathrm{~ms})$ periods of quasi-stability of the scalp electrical field and provide a measure of the momentary global state of the brain (Lehmann et al., 1987, 2009). Recently, particular microstates have been linked to activity in large-scale functional networks at rest (Britz et al., 2010; Van De Ville et al., 2010), indicating that they represent functionally different states of the mind. Also, the treatment of physically identical stimuli has been shown to vary as a function of the pre-stimulus EEG microstate (Kondakor et al., 1995; Mohr et al., 2005).

Using this analysis approach along with the intermittent paradigm, recent studies have identified two microstates during a pre-stimulus period ( -50 to $0 \mathrm{~ms}$ ) that doubly dissociate perceptual reversals from perceptual stability in the upcoming trial for both the Necker cube (Britz et al., 2009) and binocular rivalry (Britz et al., 2011). The analysis of pre-stimulus EEG microstates in this type of paradigm follows the notion that the different treatment (e.g., reversal versus stability) of physically identical stimuli arises from differences in the microstate immediately preceding 


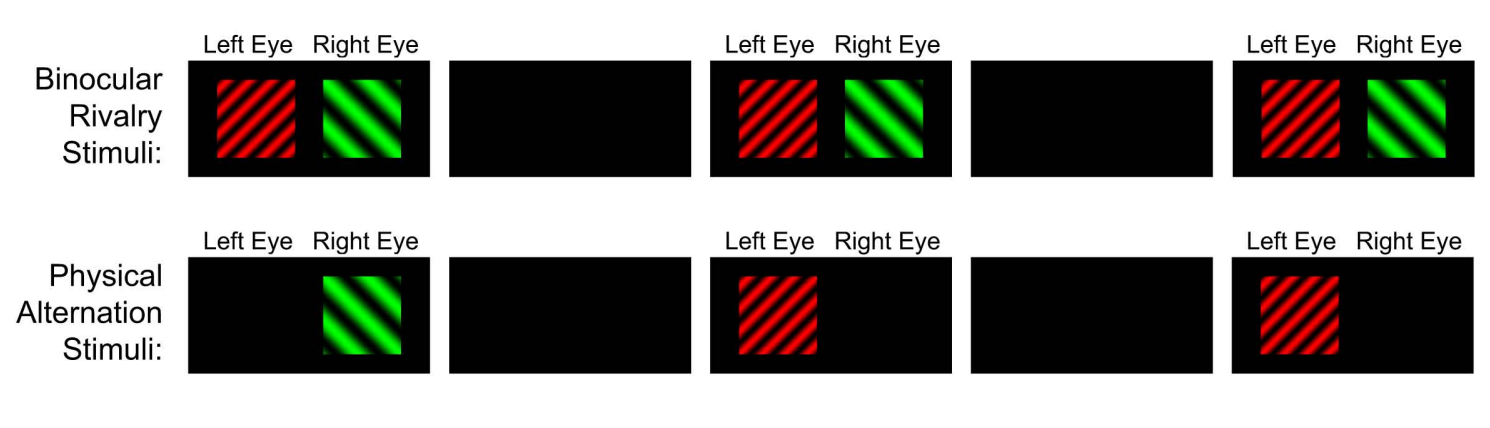

Timing:
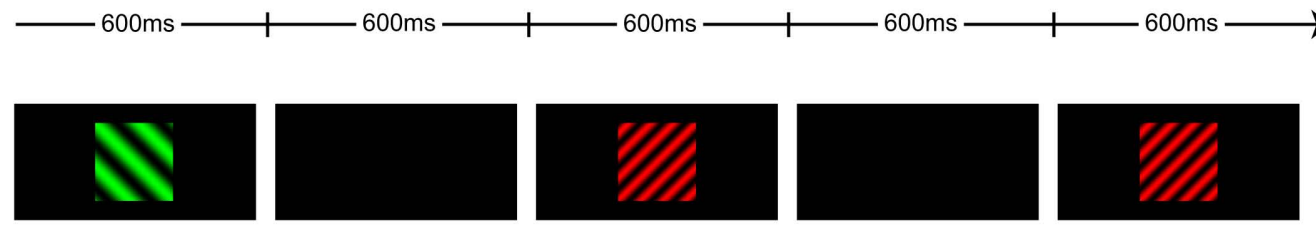

Reports:
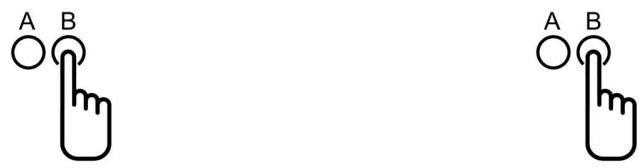

Conditions: $\quad$ Percept A

Percept B

Percept B

Reversal

Stability

FIGURE 1 | Example of the intermittent paradigm. By presenting stimuli under binocular rivalry and physical alternation conditions and instructing subjects to report their percepts on each trial, all four types of comparisons are made possible: endogenous percept $A$ versus $B$; exogenous percept $A$ versus $B$; endogenous reversal versus stability; exogenous reversal versus stability. stimulus onset. During the brief periods of quasi-stability, only the strength (i.e., the global field power, GFP), but not the configuration of the scalp electrical field can vary. Because the average duration of a microstate is $\sim 100 \mathrm{~ms}$ and it is not disrupted by the arrival of a stimulus, the GFP maximum in the 50 -ms time window prior to stimulus onset reflects the best representative sample in terms of signal-to-noise-ratio of the pre-stimulus state. In a first step, the microstates that dissociate two conditions (e.g., reversals versus stability) are identified within each subject by means of a cluster analysis. In a second step, those microstates that dissociate the conditions between subjects are likewise identified by cluster analysis.

By applying distributed source localization methods to these data, we found that the pre-stimulus activity occurring prior to perceptual reversals is likely generated in the right inferior parietal cortex (Britz et al., 2009; Britz et al., 2011). The pre-stimulus microstate associated with perceptual stability was estimated to have generators in bilateral inferior temporal (IT) areas (Britz et al., 2011). These results are in accordance with previous fMRI studies (e.g., Lumer et al., 1998) that found the right inferior parietal cortex to be involved in multistable perception, and single-cell recordings (e.g., Leopold and Logothetis, 1996) that found correlations between IT activity and the dominant percept. It is important to note however that Britz et al. (2011) found IT activity to be stronger for perceptual stability regardless of the specific percept, whereas Leopold and Logothetis (1996) correlated IT activity in distinct subsets of cells with each specific percept. Nevertheless, the combination of spatial and temporal information offered by the Electrical Neuroimaging approach allowed us to determine that these neuronal events occurred prior to perceptual reversals, thus narrowing down the possibilities for their functional contribution to binocular rivalry. Like all EEG and MEG source localization methods, distributed inverse solutions are non-unique and depend on the implemented constraints and regularization parameters. However, there is ample evidence from experimental and clinical studies showing that the constraints introduced in these distributed linear inverse solutions yield reasonable results and a spatial precision comparable to fMRI (Schulz et al., 2008; Vulliemoz et al., 2010; Grouiller et al., 2011; Laganaro et al., 2011).

Importantly, EEG microstates identified within post-stimulus time periods are consistent with traditional ERP components while eliminating potential biases inherent in choosing time windows and electrode locations for statistical tests (Michel et al., 2009). For example, we identified the RN component in a binocular rivalry experiment using both traditional ERP measures and the EEG microstate approach (Britz and Pitts, 2011). Statistical analyses in source space suggested that the $\mathrm{RN}$ is generated in inferior occipital-temporal cortex and that the scalp field during the RN time window is stronger for perceptual stability than reversals, corroborating the notion of percept stabilization in those areas (Sterzer and Rees, 2008). Similarly, the RP component was evident in both ERP and microstate analyses, and was estimated to have 
generators in extra-striate visual areas, while the LPC component appeared to be generated in superior and middle temporal as well as inferior frontal areas (Britz and Pitts, 2011).

\section{A SEQUENCE OF NEURAL EVENTS}

Based on our recent binocular rivalry experiments as well as previous ambiguous figure experiments, we can begin to map out the spatio-temporal profile of the brain events associated with bistable perception. The following model of the sequence of neural events mediating perceptual rivalry is intended to serve as an initial foundation for future investigations and is expected to be modified if warranted by additional data. Figure 2 depicts the approximate spatial locations and temporal windows in which various brain areas have been shown to be differentially activated during bistable perception. Along with the location and timing information, we have listed hypothesized functional properties as well as the names of associated ERP components (where applicable).

It is important to note that these proposed functions are based on the types of comparisons made (e.g., reversal versus stable; percept A versus B), the timing of the measured brain activity, and logical assumptions related to the temporal sequence of events. These functional accounts are intended to serve as preliminary hypotheses that are testable in future studies. For example, if the $\mathrm{RN}$ reflects the establishment of a new perceptual representation and the LPC a post-perceptual appraisal of this change, the RN should be insensitive to manipulations of task while the LPC may vary according to task demands. Similarly, it is currently unknown whether the percept A versus B effect (from 130 to $160 \mathrm{~ms}$ ) is associated with feedback from higher to lower level visual areas and whether the timing of this effect depends on the particular type of perceptual competition involved.

Overall, work spanning the past 5-10 years has produced converging evidence regarding the timing and spatial locations of the neural events involved in bistable perception. Now that the temporal dynamics of these components and their intracranial sources have been identified and have been shown to be robust and reliable, more fine-tuned manipulations are necessary to reveal the precise functional significance of each component. It is our hope that this model will serve as a decent starting point.

\section{BINOCULAR RIVALRY AND PERCEPTUAL AWARENESS}

In our spatio-temporal model (Figure 2) we assume that the pre-stimulus inferior parietal activity as well as the post-stimulus extra-striate activity (RP) are not directly linked with perceptual awareness. To become aware of a stimulus, the stimulus must be present, so activity preceding stimulus onset may influence but is unlikely to directly reflect perceptual awareness. The timing of the RP (100-130 ms) was found to precede the signal that diverged according to the reported percept (130-160 ms), and is thus similarly unlikely to index awareness. The delayed activity in anatomically early visual areas (130-160 ms), however, varied according to perception during rivalry and is therefore a candidate neural correlate of perceptual awareness. Alternatively, this delayed activity might index a non-conscious (or preconscious) stage of processing in which the competition between percepts is resolved but requires an interaction with higher-level visual areas to enable the formation of a stable perceptual representation. In

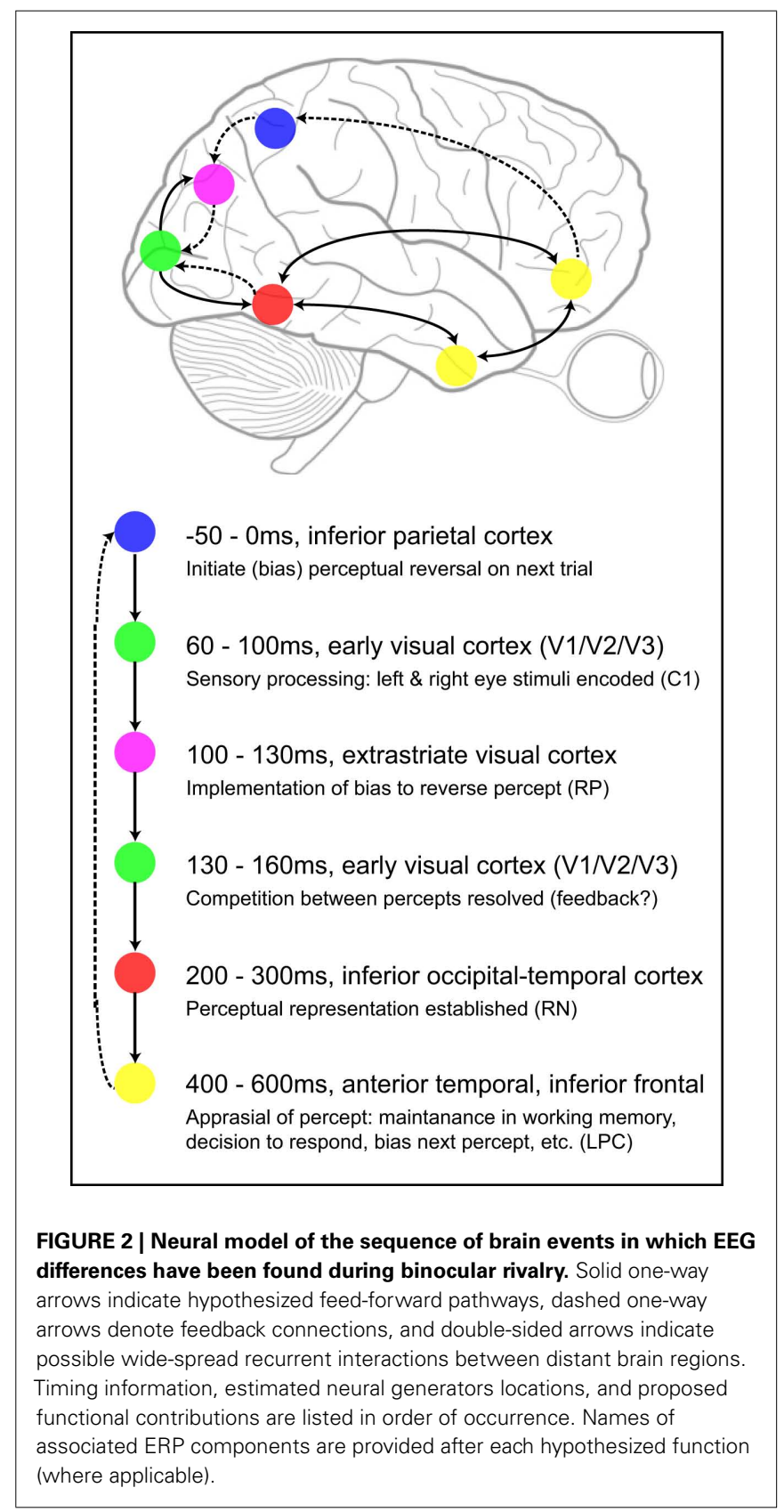

this latter view, the RN component (at 200 to $300 \mathrm{~ms}$, with generators in inferior occipital-temporal cortex) would become the primary candidate for a neural correlate of awareness. Finally, while unlikely, the RN might index the perceptual change preconsciously while the LPC (with its wide-spread cortical sources) might reflect conscious processing of the perceptual information or maintenance of the percept in working memory. Alternatively, the occipital-temporal activity reflected by the RN may be more closely associated with what some theorists (e.g., Block, 2005) have dubbed "phenomenal consciousness," while the wide-spread activity indexed by the LPC may represent "access consciousness."

A notable finding with implications for perceptual awareness has been the striking similarity between ERPs/microstates 
identified for binocular rivalry and those identified for ambiguous figures (Britz et al., 2009; Britz and Pitts, 2011). It is often assumed that while perceptual alternations exhibit similar characteristics (e.g., reversal rates) in both cases, the underlying neural mechanisms, especially those contributing to perceptual competition and resolution, must be different. However, as suggested more than 10 years ago (Leopold and Logothetis, 1999), at least some of the neural networks involved may be similar across all types of bistable perception and these networks may reside in non-visual areas. The pre-stimulus (microstates from -50 to $0 \mathrm{~ms}$ ) and postperceptual (LPC) effects described above are consistent with this hypothesis in that their estimated generators are located in frontalparietal areas. Recently, transcranial magnetic stimulation (TMS) has been employed to test the causal role of frontal-parietal areas in initiating perceptual reversals (Kanai et al., 2010; Zaretskaya et al., 2010). Interestingly, disruption of activity via TMS in different subregions of the parietal cortex appears to result in opposite effects (increasing or decreasing reversal rates), thus suggesting that a more complex network of parietal regions is involved in bistable perception (Kanai et al., 2011). Inferior frontal regions have also been implicated as playing a role in the initiation of perceptual reversals (Sterzer and Kleinschmidt, 2007). In a recent study, TMS applied over frontal areas was found to slow perceptual reversals, but only when reversals were under voluntary control (de Graaf et al., 2011). This finding is consistent with an earlier study that found reversal rates in frontal lesion patients to be normal during passive viewing, but impaired during a voluntary reversal condition (Windmann et al., 2006). Clearly frontal-parietal areas are involved in the initiation of perceptual reversals, but the precise contribution of each region (and possibly different subregions) appears to be complex and warrants further investigation.

\section{REFERENCES}

Blake, R., and Logothetis, N. K. (2002). Visual competition. Nat. Rev. Neurosci. 3, 13-21.

Block, N. (2005). Two neural correlates of consciousness. Trends Cogn. Sci. 9 , 46-52.

Brascamp, J. W., Pearson, J., Blake, R., and van den Berg, A. V. (2009). Intermittent ambiguous stimuli: implicit memory causes periodic perceptual alternations. J. Vis. 9, 3, 1-23.

Britz, J., Landis, T., and Michel, C. M. (2009). Right parietal brain activity precedes perceptual alternation of bistable stimuli. Cereb. Cortex 19, 55-65.

Britz, J., and Pitts, M. A. (2011). Perceptual reversals during binocular rivalry: ERP components and their concomitant source differences. Psychophysiology. doi: 10.1111/j.14698986.2011.01222.x. [Epub ahead of print].

Britz, J., Pitts, M. A., and Michel, C. M. (2011). Right parietal brain activity precedes the perceptual reversals during binocular rivalry. Hum. Brain Mapp. 32, 1432-1442.
Britz, J., Van De Ville, D., and Michel, C. M. (2010). BOLD correlates of EEG topography reveal rapid restingstate network dynamics. Neuroimage 52, 1162-1170.

de Graaf, T., de Jong, M., Goebel, R., van Ee, R., and Sack, A. (2011). On the functional relevance of frontal cortex for passive and voluntarily controlled bistable vision. Cereb. Cortex 21, 2322-2331.

Grouiller, F., Thornton, R. C., Groening, K., Spinelli, L., Duncan, J. S., Schaller, K., Siniatchkin, M., Lemieux, L., Seeck, M., Michel, C. M., and Vulspikes: localization of focal epileptic activity by simultaneous electroencephalography and functional magnetic resonance imaging. Brain. doi:10.1093/brain/awr156. [Epub ahead of print].

Intaite, M., Koivisto, M., Ruksenas, O., and Revonsuo, A. (2010). Reversal negativity and bistable stimuli: attention, awareness, or something else? Brain Cogn. 74, 24-34.

Kanai, R., Bahrami, B., and Rees, G. (2010).Human parietal cortex strucliemoz, S. (2011). With or without

Interestingly, the $\mathrm{RN}$ component also appears to be invariant with respect to the type of bistability involved. One possibility is that the neural mechanisms supporting the initiation and appraisal of perceptual reversals are common for all types of bistable figures, while the intermediate stages supporting stimulus/percept competition and resolution vary according to the particular stimuli involved. In this view, the RN would reflect a post-perceptual stage of processing, while earlier activity (e.g., the delayed response in V1/V2/V3) would index the perceptual representation. Future experiments may be able to test this hypothesis by systematically varying the competing features of the stimuli (e.g., orientation, color, motion, shape, etc.) to determine which $\mathrm{ERP} /$ microstate varies according to the specific features of the stimuli and thus most closely reflect the contents of perceptual awareness.

While binocular rivalry (and ambiguous figures) are undoubtedly powerful vehicles for dissociating sensory input from perceptual experience, their potential for helping determine the neural basis of perceptual awareness may be truncated by more direct manipulations of awareness. For each type of comparison outlined above (e.g., percept A versus B, reversals versus stability) subjects are always aware of one of the two stimuli/percepts. Thus, a straight-forward comparison between "aware" and "unaware" conditions is not possible. Other types of paradigms, such as the attentional blink (Sergent et al., 2005), inattentional blindness (Pitts et al., 2011), and backward masking (Koivisto and Revonsuo, 2010) may be more appropriate when the goal is to identify neural events linked with conscious perception. Nevertheless, binocular rivalry has proven to be largely successful in separating sensory input from subjective perception and will undoubtedly continue to be a valuable tool as our methodological techniques are further refined.

ture predictsindividual differences in perceptual rivalry. Curr. Biol. 20, 1626-1630.

Kanai, R., Carmel, D., Bahrami, B., and Rees, G. (2011). Structural and functional fractionation of right superior parietal cortex in bistable perception. Curr. Biol. 21, R106-R107.

Koivisto, M., and Revonsuo, A. (2010). Event-related brain potential correlates of visual awareness. Neurosci. Biobehav. Rev. 34, 922-934.

Kondakor, I., Pascual-Marqui, R. D., Michel, C. M., and Lehmann, D. (1995). Event-related potential map differences depend on the prestimulus microstates. J. Med. Eng. Technol. 19, 66-69.

Kornmeier, J., and Bach, M. (2004). Early neural activity in Necker-cube reversal: evidence for low-level processing of a gestalt phenomenon. Psychophysiology 41, 1-8.

Kornmeier, J., and Bach, M. (2005). The Necker cube - an ambiguous figure disambiguated in early visual processing. Vision Res. 45, 955-960.

Kornmeier, J., Ehm, W., Bigalke, H., and Bach, M. (2007). Discontinuous presentation of ambiguous figures: how interstimulus-interval durations affect reversal dynamics and ERPs. Psychophysiology 44, 552-560.

Laganaro, M., Morand, S., Michel, C. M., Spinelli, L., and Schnider, A. (2011). ERP correlates of word production before and after stroke in an aphasic patient. J. Cogn. Neurosci. 23, 374-381.

Lehmann, D., Ozaki, H., and Pal, I. (1987). EEG alpha map series: brain micro-states by space-oriented adaptive segmentation. Electroencephalogr. Clin. Neurophysiol. 67, 271-288.

Lehmann, D., Pascual-Marqui, R. D., and Michel, C. M. (2009). EEG microstates. Scholarpedia 4, 7632.

Leopold, D. A., and Logothetis, N. K. (1996). Activity changes in early visual cortex reflect monkeys' percepts during binocular rivalry. Nature 379, 549-553.

Leopold, D. A., and Logothetis, N. K. (1999). Multistable phenomena: changing views in perception. Trends Cogn. Sci. 3, 254-264. 
Leopold, D. A., Wilke, M., Maier, A., and Logothetis, N. K. (2002). Stable perception of visually ambiguous patterns. Nat. Neurosci. 5, 605-609.

Logothetis, N. K., Leopold, D. A., and Sheinberg, D. L. (1996). What is rivalling during binocular rivalry? Nature 380, 621-624.

Lumer, E. D., Friston, K. J., and Rees, G. (1998). Neural correlates of perceptual rivalry in the human brain. Science 280, 1930-1934.

Lumer, E. D., and Rees, G. (1999). Covariation of activity in visual and prefrontal cortex associated with subjective visual perception. Proc. Natl. Acad. Sci. U.S.A. 96, 1669-1673.

Michel, C. M., Koenig, T., Brandeis, D., Gianotti, L., and Wackermann, J. (2009). Electrical Neuroimaging. Cambridge: Cambridge University Press.

Mohr, C., Michel, C. M., Lantz, G., Ortigue, S., Viaud-Delmon, I., and Landis, T. (2005). Brain state-dependent functional hemispheric specialization in men but not in women. Cereb. Cortex 15, 1451-1458.

O'Donnell, B. F., Hendler, T., and Squires, N. K. (1988). Visual evoked potentials to illusory reversals of the Necker cube. Psychophysiology 25, 137-143.

Orbach, J., Ehrlich, D., and Heath, H. (1963). Reversibility of the
Necker cube: I. An examination of the concept of "satiation of orientation.” Percept. Mot. Skills 17, 439-458.

Orbach, J., Zucker, E., and Olson, R. (1966). Reversibility of the Necker cube: VII. Reversal rate as a function of figure-on and figure-off durations. Percept. Mot. Skills 22, 615-618.

Pitts, M. A., Martínez, A., and Hillyard, S. A. (2010). When and where is binocular rivalry resolved in the visual cortex? J. Vis. 10, 1-11.

Pitts, M. A., Martínez, A., and Hillyard, S. A. (2011). Visual processing of contour patterns under conditions of inattentional blindness. J. Cogn. Neurosci. doi: 10.1162/jocn_a_00111. [Epub ahead of print].

Pitts, M. A., Martínez, A., Stalmaster, C., Nerger, J. L., and Hillyard, S. A. (2009). Neural generators of ERPs linked with Necker cube reversals. Psychophysiology 46, 694-702.

Pitts, M. A., Nerger, J. L., and Davis, T. J. R. (2007). Electrophysiological correlates of perceptual reversals for three different types of multistable images. J. Vis. 7, 1-14.

Schulz, E., Maurer, U., Van Der Mark, S., Bucher, K., Brem, S., Martin, E., and Brandeis, D. (2008). Impaired semantic processing during sentence reading in children with dyslexia: combined fMRI and ERP evidence. Neuroimage 41, 153-168.

Sergent, C., Baillet, S., and Dehaene, S. (2005). Timing of the brain events underlying access to consciousness during the attentional blink. Nat. Neurosci. 8, 1391-1400.

Sterzer, P., and Kleinschmidt, A. (2007). A neural basis for inference in perceptual ambiguity. Proc. Natl. Acad. Sci. U.S.A. 104, 323-328.

Sterzer, P., Kleinschmidt, A., and Rees, G. (2009). The neural bases of multistable perception. Trends Cogn. Sci. 13, 310-318.

Sterzer, P., and Rees, G. (2008). A neural basis for percept stabilization in binocular rivalry. J. Cogn. Neurosci. 20, 389-399.

Tong, F., Meng, M., and Blake, R. (2006). Neural bases of binocular rivalry. Trends Cogn. Sci. 10, 502-511.

Van De Ville, D., Britz, J., and Michel, C. M. (2010). EEG microstate sequences in healthy humans at rest reveal scale-free dynamics. Proc. Natl. Acad. Sci. U.S.A. 107, 18179-18184.

Vulliemoz, S., Rodionov, R., Carmichael, D. W., Thornton, R., Guye, M., Lhatoo, S. D., Michel, C. M., Duncan, J. S., and Lemieux, L. (2010). Continuous EEG source imaging enhances analysis of EEG-fMRI in focal epilepsy. Neuroimage 49, 3219-3229.
Windmann, S., Wehrmann, M., Calabrese, P., and Gunturkun, O. (2006). Role of the prefrontal cortex in attentional control over bistable vision. J. Cogn. Neurosci. 18, 456-471.

Zaretskaya, N., Thielscher, A., Logothetis, N. K., and Bartels, A. (2010). Disrupting parietal function prolongs dominance durations in binocular rivalry. Curr. Biol. 20, 2106-2111.

Conflict of Interest Statement: The authors declare that the research was conducted in the absence of any commercial or financial relationships that could be construed as a potential conflict of interest.

Received: 24 June 2011; paper pending published: 20 July 2011; accepted: 11 September 2011; published online: 29 September 2011.

Citation: Pitts MA and Britz J (2011) Insights from intermittent binocular rivalry and EEG. Front. Hum. Neurosci. 5:107. doi: 10.3389/fnhum.2011.00107 Copyright (c) 2011 Pitts and Britz. This is an open-access article subject to a nonexclusive license between the authors and Frontiers Media SA, which permits use, distribution and reproduction in other forums, provided the original authors and source are credited and other Frontiers conditions are complied with. 